\title{
Association Between Vitamin E and Handgrip Strength in the Korean General Population in KNHANES VII (2018)
}

\author{
Nodam Park, $\mathrm{MD}^{1}$, Soo A Kim, MD, $\mathrm{PhD}^{1}$, Kiyoung $\mathrm{Oh}, \mathrm{MD}, \mathrm{PhD}^{1}$, Yuntae Kim, $\mathrm{MD}^{1}$, \\ Siha Park, $\mathrm{MD}^{1}$, Joon Yeop Kim, $\mathrm{MD}^{1}$, Namhun Heo, $\mathrm{MS}^{2}$ \\ ${ }^{1}$ Department of Physical Medicine and Rehabilitation, Soonchunhyang University Cheonan Hospital, \\ Soonchunhyang University College of Medicine, Cheonan; \\ ${ }^{2}$ Clinical Trial Center, Soonchunhyang University Cheonan Hospital, Cheonan, Korea
}

Objective To investigate the association between vitamin E and handgrip strength (HGS) with multiple factors. Methods A total of 1,814 participants were included ( 822 men and 981 women) from the Korean subjects of the 7th Korea National Health and Nutrition Examination Survey in 2018. Data were analyzed using multiple logistic regression to determine the correlation between vitamin E and HGS with potential confounding factors.

Results In the multiple logistic regression model, only the young age group (19-40 years) of men showed a positive relationship between vitamin E and HGS. However, in older age groups (41-80 years) of men and all age groups of women, there was no statistically significant result. After adjusting for confounding factors, young men showed higher vitamin E levels and higher HGS. Conversely, women and older age groups did not show significant results after adjusting for confounding factors.

Conclusion In this study, the serum vitamin E level had a positive effect on HGS in young men ( $<40$ years). Further research is needed on this topic regarding vitamin $\mathrm{E}$ intake and other objective measures.

Keywords Handgrip strength, Vitamin E, KNHANES VII, Korea

\section{INTRODUCTION}

Diet and exercise are essential for maintaining health in an aging society. The loss of muscle strength and mass in the elderly, known as sarcopenia, is considered an inde- pendent risk factor for falls, fragility, disability, and lower quality of life. Handgrip strength (HGS) has been used as an important index of muscle strength to diagnose sarcopenia because low HGS is a clinical marker of poor mobility and low muscle mass. Because it has low cost and

Received February 18, 2021; Revised March 23, 2021; Accepted March 31, 2021; Published online June 14, 2021

Corresponding author: Soo A Kim

Department of Physical Medicine and Rehabilitation, Soonchunhyang University Cheonan Hospital, Soonchunhyang University College of Medicine, 31 Suncheonhyang 6-gil, Dongnam-gu, Cheonan 31151, Korea. Tel: +82-41-570-3874, Fax: +82-41-570-2776, E-mail: sooapmr@schmc.ac.kr ORCID: Nodam Park (https://orcid.org/0000-0002-2871-0202); Soo A Kim (https://orcid.org/0000-0003-1578-0452); Kiyoung Oh (https://orcid. org/0000-0002-1886-5462); Yuntae Kim (https://orcid.org/0000-0003-4063-4692); Siha Park (https://orcid.org/0000-0002-8673-1140); Joon Yeop Kim (https://orcid.org/0000-0002-8570-8336); Namhun Heo (https://orcid.org/0000-0001-7278-2214).

(c) This is an open-access article distributed under the terms of the Creative Commons Attribution Non-Commercial License (http://creativecommons.org/ licenses/by-nc/4.0) which permits unrestricted noncommercial use, distribution, and reproduction in any medium, provided the original work is properly cited. Copyright $\odot 2021$ by Korean Academy of Rehabilitation Medicine 
is easy to measure and perform at bedside, HGS also has been used widely in previous studies focused on its association with the risk of cardiovascular disease, cancer, and cognitive impairment, as well as sarcopenia.

Several studies have been conducted to identify other factors that contribute to muscle strength. Nutrition is one of the major factors that can be modified. Several studies have reported that nutritional supplementation with proteins, amino acids, vitamin $\mathrm{D}$, calcium, and other antioxidants can improve muscle strength. For example, vitamin D is well known for its antioxidant activity against free radical damage and plays a major role in protein synthesis through vitamin D receptors (VDR) in muscle, which improves muscle strength and physical function $[1,2]$. Various cross-sectional studies have reported a positive relationship between vitamin $\mathrm{D}$ levels and muscle strength. Thus, vitamin D deficiency may be a risk factor for sarcopenia in elderly people $[1,2]$. Recently, some preclinical and human experimental studies have shown that vitamin $\mathrm{E}$, as an antioxidant, promotes myoblast proliferation and increases muscle mass [3]. A previous study also found that higher tocopherol levels were associated with greater muscle strength measures, such as handgrip and knee strength in elderly women and a lower tendency of fragility in elderly women and men [4].

Recently, vitamin $\mathrm{E}$ has attracted public attention as a key nutrient in the defensive role of antiaging $[5,6]$. Vitamin E, which consists of two subgroups (tocopherol and tocotrienol), has antioxidant and anti-inflammatory properties that result in antiaging effects [3,7]. It is a lipidsoluble vitamin that plays the role of an antioxidant agent that scavenges reactive oxygen species in the skeletal muscle and is vulnerable to oxidative damage because of its high oxygen consumption [5,7]. Vitamin E can reduce muscle damage, enhance recovery from exercise, and prevent muscle atrophy [8]. Therefore, there has been growing interest in the effect of vitamin $\mathrm{E}$ on enhancing muscle strength and exercise performance because vitamin $\mathrm{E}$ plays a major role in reducing muscle fatigue. Several in vivo and in vitro studies have demonstrated that supplementation with vitamin E could be beneficial for decreasing oxidation [9], suppressing oxidative stress, increasing protein synthesis [10], and reducing lipid peroxidase [6] as an oxidative stress biomarker against free radical assault during exercise [3].
Although there is much evidence suggesting an association between vitamin $\mathrm{D}$ and muscle strength, few studies have investigated the association between vitamin $\mathrm{E}$ and muscle strength. Therefore, this study was performed to evaluate the association between vitamin $\mathrm{E}$ and HGS after adjusting for multiple confounding factors. We used cross-sectional data from the population-based Korea National Health and Nutrition Examination Survey (KNHANES) in Korean adults aged $\geq 19$ years.

\section{MATERIALS AND METHODS}

\section{Participants}

This study was based on data from the Korean subjects of the 7th Korea National Health and Nutrition Examination Survey (KNHANES VII) in 2018. The total number of participants in KNHANES VII was 7,992. This study included patients aged $\geq 19$ years $(n=6,489)$. We excluded participants with missing values for serum vitamin $\mathrm{E}$ levels $(n=4,675)$. The total number of study subjects was 1,814 (833 men and 981 women) (Fig. 1). The subjects were divided into three groups: young age group (1940 years), middle age group (41-64 years), and elderly age group (65-80 years). All participants provided written informed consent.

\section{Handgrip strength}

In KNHANES VII, the HGS test was used to determine the distribution of muscle strength. HGS was measured using a digital handgrip dynamometer (digital grip strength dynamometer, TKK 5401; Takei, Niigata, Japan). The participants were asked which hand was the dominant hand. This test was repeated three times with both

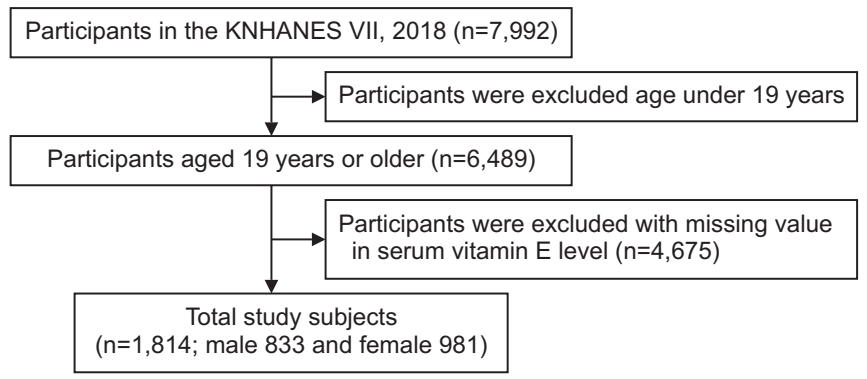

Fig. 1. The participant flow diagram is shown. KNHANES VII, 7th Korea National Health and Nutrition Examination. 
dominant and non-dominant hands alternately, and we selected the maximal HGS among the data.

\section{Vitamin $\mathrm{E}$}

Vitamin $\mathrm{E}$ was included in the survey from KNHANES VII (2016-2018). Vitamin E (mg/L) was detected from the sample by Agilent 1200 (Agilent Technologies Inc., Santa Clara, CA, USA) tools and Chromsystems (Gräfelfing, Germany) reagent through high-performance liquid chromatography with flame ionization detector methods.

\section{Assessment of covariates}

Every subject aged $\geq 19$ years was asked to complete a health questionnaire to obtain information about age, sex, household income, alcohol consumption, smoking status, daily nutrition intake, aerobic physical activity level, and so on. According to drinking experience in the patient's lifetime and drinking frequency for the past year, participants were divided into three groups: nondrinkers who had never consumed alcohol, ex-drinkers who had not consumed alcohol in the past, and current drinkers. Smoking status was classified into three groups based on current smoking status (non-smoker, ex-smoker, and current smoker). Daily energy and nutritional intake were investigated using food frequency questionnaires (FFQs). Aerobic physical activity was defined as "yes" for those who had performed medium-intensity exercise for at least 2 hours and 30 minutes per week or high-intensity exercise for least 1 hour and 15 minutes or a combination of medium- and high-intensity exercise ( 1 minute of high intensity $=2$ minutes of medium intensity). Body mass index (BMI, $\mathrm{kg} / \mathrm{m}^{2}$ ) was calculated by measuring body weight and height according to standardized procedures for all participants.

\section{Statistical analyses}

This study used raw data from KNHANES VII conducted in 2018. We used the integrated weights suggested in the guidelines for analyzing the raw data from the National Health and Nutrition Survey and analyzed it by considering the effect of the complex sample design.

Differences in general characteristics according to age and sex were compared using Student t-test or the chisquare test, as appropriate for the entire sample and age groups (young age, 19-39 years; middle age, 40-64 years; elderly age, 65-80 years). Student t-test was performed to determine the mean \pm standard error for continuous variables such as age, nutrition intake, HGS, and so on. The chi-square test was performed to determine $\mathrm{n}(\%)$ for categorical variables such as household income, alcohol consumption, smoking status, presence or absence of depression, aerobic physical activity level, and so on. Multiple linear regression analysis was performed to examine whether there was an independent relationship between HGS and vitamin $\mathrm{E}$ in the entire sample and age groups based on sex. We used confounding factors with proven associations with sarcopenia in a previous study. Models 1 (without adjusting for confounding factors), model 2 (adjusted for age and BMI), and model 3 (adjusted for age, BMI, household income, alcohol consumption, smoking status, aerobic physical activity level, daily energy, and nutrition intake [carbohydrate, fat, and protein]) were applied.

All statistical analyses were performed using SPSS version 26.0 (IBM SPSS, Armonk, NY, USA) and statistical significance was set at $\leq 0.05$.

\section{RESULTS}

The total number of subjects was 1,814: 883 (45.92\%) men and 981 (54.08\%) women. All subjects were divided into three groups according to age (young age group [1939 years], $\mathrm{n}=636$; middle age group [40-64 years], $\mathrm{n}=872$; elderly age group [65-80 years], $n=306$ ). Demographic characteristics by sex and age group are shown in Table 1 . There were significant differences in most of the parameters among the groups, except household income by gender, carbohydrate intake by age, and vitamin $\mathrm{E}$ by age and sex. Men had greater HGS than women; the mean HGS of men was $39.17 \mathrm{~kg}$, and that of women was $22.86 \mathrm{~kg}$. The young age group tended to have greater HGS: young age group, $33.06 \mathrm{~kg}$; middle age group, $31.40 \mathrm{~kg}$; and elderly age group, $25.24 \mathrm{~kg}$. The mean serum vitamin E levels were 12.56 and $13.28 \mathrm{mg} / \mathrm{L}$ in men and women, respectively. The young age group showed a lower vitamin $\mathrm{E}$ level of $11.38 \mathrm{mg} / \mathrm{L}$. The middle and elderly age groups had similar values: 13.80 and $13.86 \mathrm{mg} / \mathrm{L}$, respectively.

We analyzed the relationship between vitamin $\mathrm{E}$ and HGS using multiple linear regression according to age and sex. There were three models. Model 1 showed a relationship without correction for any other confounding factors. Model 2 was a minimally adjusted model 


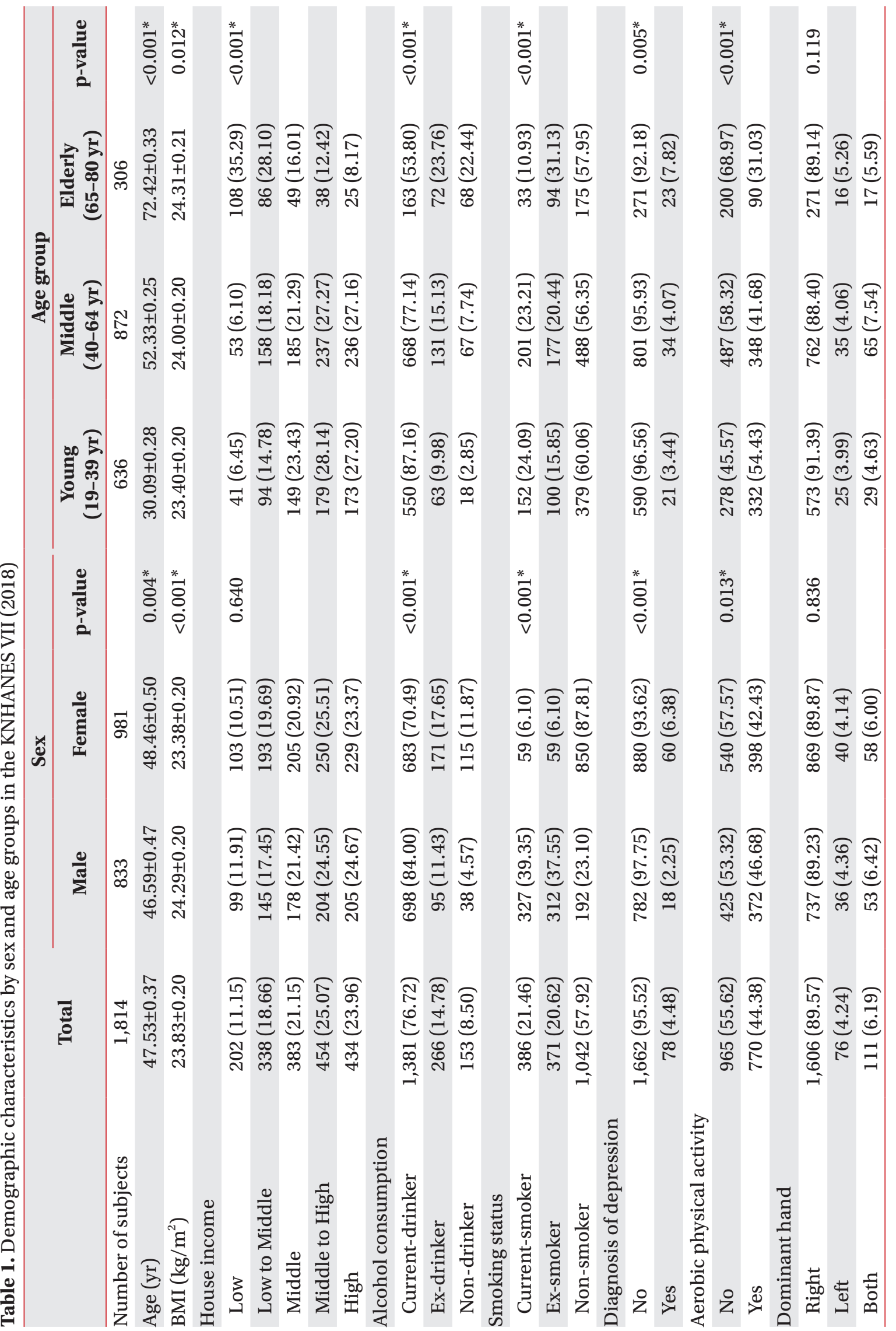



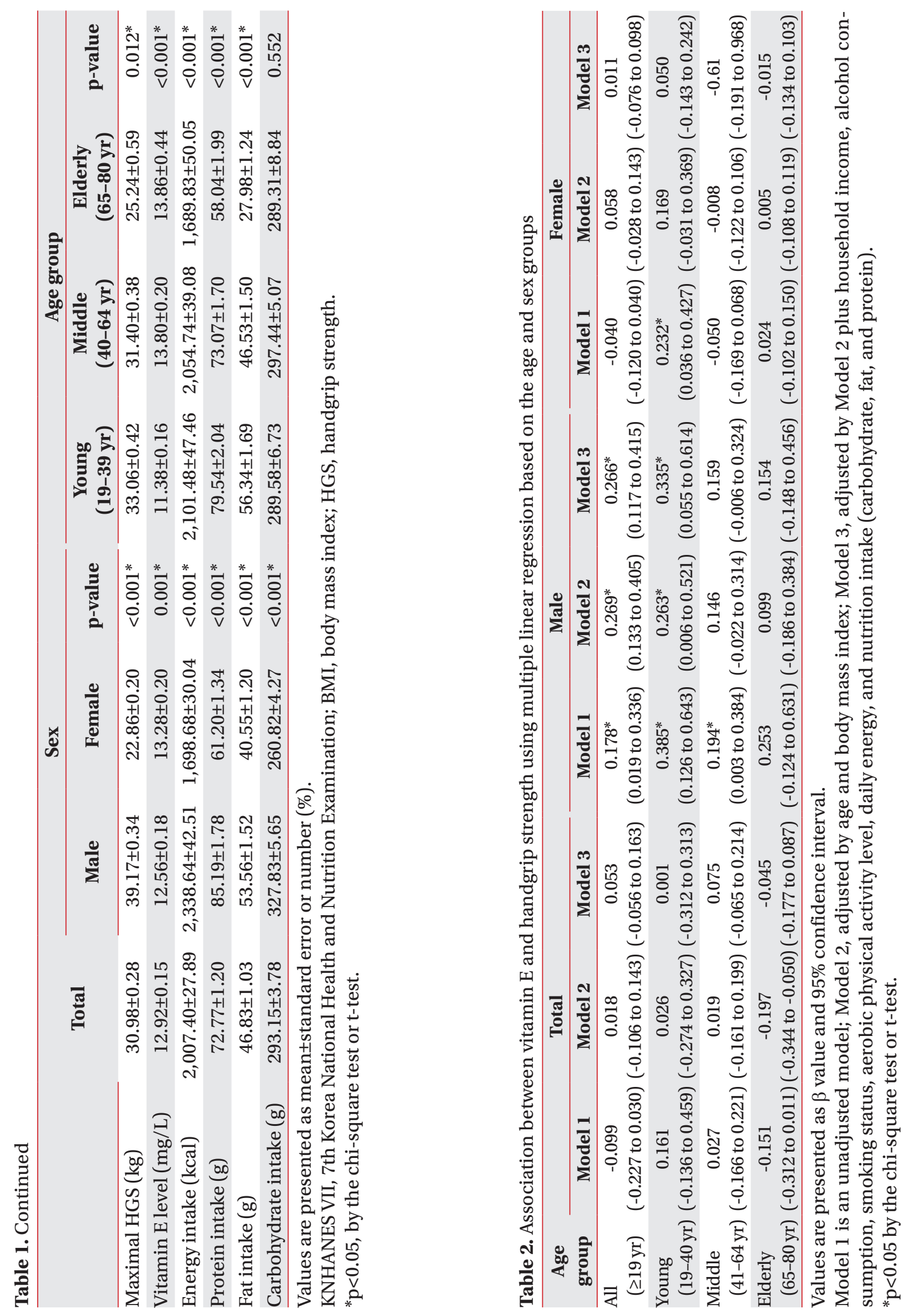
based on age and BMI. Model 3 was adjusted for age, BMI, household income, alcohol consumption, smoking status, aerobic physical activity level, daily energy, and nutrition intake (carbohydrate, fat, and protein).

Table 2 shows the association between vitamin $\mathrm{E}$ and HGS based on age and sex. In all subjects combined men and women, there was no significant association between vitamin $\mathrm{E}$ and HGS in all of the models, except model 2 in the elderly age group ( $p=0.009)$. In the male group, regardless of confounding factors, the serum vitamin E level had a positive relationship with HGS in all age groups ( $\mathrm{p}=0.028$ in model $1 ; \mathrm{p}<0.001$ in models 2 and 3 ) and the young age group ( $\mathrm{p}=0.004,0.045$, and $0.019 \mathrm{in}$ models 1,2 , and 3, respectively). However, in the middle age group, only model 1 showed statistically significant results $(p=0.046)$. Even in the elderly age group, there were no significant results in any of the three models. In the female group, there were no significant results except for model 1 in the young age group $(p=0.021)$.

\section{DISCUSSION}

In this study, we analyzed the correlation between vitamin E and HGS in Korean adults aged $\geq 19$ years. Only men showed higher levels of HGS with higher vitamin $\mathrm{E}$ levels, especially in the young age group. However, there were no significant results in women and all subjects combined (men and women). In particular, in the elderly group, there was no significant correlation even in men.

There have been several studies on the association between vitamin $\mathrm{E}$ and muscle strength. Most previous studies were cross-sectional studies conducted in the elderly. A previous study demonstrated positive relationships between $\alpha$-tocopherol and muscle strength, including handgrip, hip flexion, and knee extension power, in 669 older women living in the community aged $\geq 70$ years [4]. Vitamin E was divided into quartiles, and handgrip, hip, and knee strength were divided into tertiles, without widely accepted cutoff points to define low vitamin $\mathrm{E}$ level or muscle weakness in older women. Another study suggested that in both elderly men and women aged $\geq 65$ years, highly frail participants represented by selfreported weight loss, low energy, slow gait speed, low physical activity, and low HGS showed low levels of ageand gender-adjusted vitamin E levels [11]. However, the two studies mentioned earlier were unable to explain the continuous correlation between vitamin $\mathrm{E}$ and muscle strength because they analyzed categorized consecutive variables without a definite cutoff value. There was also an intervention study that supplemented vitamin E, but this was not a cross-sectional study. Bo et al. [12] analyzed 60 subjects with sarcopenia aged 60-85 years who underwent a double-blind, randomized, placebo-controlled trial. They showed that nutritional supplementation consisting of high whey protein and vitamins $\mathrm{D}$ and E improves the relative skeletal mass index measured by bioimpedance analysis, HGS, and physical function including gait speed after a 6-month intervention compared with the placebo group. However, they conducted interventions using not only single nutrition but also multiple nutrition, so there was a limitation in analyzing the effect of vitamin E alone on muscle strength.

The human body is composed of two types of muscle fibers according to the contraction speed, and muscle fibers have different metabolic oxidation [13], which may play different roles along with vitamin E [8]. Type I muscle fiber is a slow-twitching red muscle and has a large amount of myoglobin and capillary contents, which contribute greatly to oxidative capacity [13]. Thus, type I muscle fibers tend to use more vitamin $\mathrm{E}$ than type II muscle fibers [8]. However, a previous study showed that although type I fibers largely depend on oxidative metabolism, type II fibers generate more free radicals $[3,14]$. Several studies strongly suggest that the antioxidant and anti-inflammatory properties of vitamin E may reduce damage to type II fibers and relieve type I contractile dysfunction $[3,6]$. Therefore, the distribution of muscle fibers may be important in determining the effect of vitamin $\mathrm{E}$ on muscle.

The distribution of muscle fibers may change depending on sex or age. According to previous studies, aging induces loss of muscle fiber number as well as muscle atrophy, which are the principal causes of sarcopenia $[3,14,15]$. The effects of aging on muscle fiber loss contributed equally regardless of muscle fiber type [16], but several studies have demonstrated that type II muscle fibers are more affected than type I muscle fibers in the case of muscle atrophy $[3,14,15]$. Therefore, the distribution of type I muscle fibers was higher than that of type II fibers in the elderly. Some studies have analyzed the distribution and contractile properties, such as crosssectional area (CSA), maximal isometric force, shorten- 
ing velocity, and specific force of muscle fibers according to sex $[17,18]$. Most studies have shown that type II fibers are larger in men, while type I fibers tend to be larger in women $[17,19]$. CSA was smaller in women than in men; especially, the loss of type II area was more dominant in women $[17,20]$, which means that the proportion of type I fibers might be larger in women than in men. Among the contractile properties, type II fiber had a higher value than type I in both men and women, but there was no evidence of gender-related differences [17].

Since the effect of vitamin E on muscle is based on a defensive mechanism against metabolic catabolism after exercise [3], the intensity and duration of aerobic physical activity might be important for the role of vitamin E. Our results showed that men and the young age group performed aerobic exercise more than women and the older age groups, respectively (Table 1). It seems that the skeletal muscle may be more sensitive to changes in vitamin E levels in younger men with relatively more aerobic exercise.

Our results showed a significant correlation in men, especially at a young age. However, there were no significant results in the elderly, who were expected to be more affected, nor in women.

Previous studies have shown that poor vitamin E status increases the risk of cardiovascular disease, diabetes mellitus, and cancer. HGS was also affected by such diseases, and sarcopenia comorbidity could negatively affect HGS. We did not adjust for such comorbidities, so this might be one of the reasons for the unexpected results. Other dietary antioxidants, such as vitamins $\mathrm{C}$ and $\mathrm{D}$ and calcium, were not included in the present study and should be addressed in future investigations.

The KNHANES did not include dietary intake data of vitamin E, which is one of the potential limitations of the present study. However, assessing dietary vitamin $\mathrm{E}$ intake using FFQs is difficult [21] because the source of oil used in food is often not listed, which means vitamin E status from dietary surveys has measurement error. Furthermore, a previous study using the third National Health and Nutrition Examination Survey of the United States showed that the 24-hour dietary recall was not well correlated with serum concentrations [22]. Since this study was a cross-sectional study without a control group, the expected quantitative relationship might not be obtained. Thus, a randomized controlled study is needed to investigate whether vitamin E supplementation could improve contractile properties in the elderly, especially in patients with sarcopenia.

HGS may not be sufficient to represent total muscle strength; therefore, other body composition should be considered. In addition, considering defensive mechanism against free radial after exercise, it is necessary to analyze relationship between vitamin $\mathrm{E}$ and another objective measures, such as biomarkers that indicate oxidative stress and skeletal muscle protein turnover or exercise-induced muscle fatigue.

In conclusion, to the best of our knowledge, this is the first study to suggest a continuous correlation between serum vitamin E levels and HGS in Korean adults, from the young and middle ages to the elderly, that is related to sarcopenia using KNHANES VII in 2018 in Korea. There was no statistically significant correlation between vitamin E and HGS, except for young men with higher serum vitamin $\mathrm{E}$ levels showing higher HGS. In particular, contrary to our expectations, the correlation was not significant in the elderly.

We suggest that vitamin $\mathrm{E}$ is correlated with HGS as an indicator of sarcopenia; however, further investigations should be considered. We recommend the need for further research to analyze the relationship between vitamin E intake and objective measures such as contractile properties and physical performance. In addition, it is necessary to investigate the role of vitamin E depending on muscle type by age and sex.

\section{CONFLICT OF INTEREST}

No potential conflict of interest relevant to this article was reported.

\section{ACKNOWLEDGMENTS}

The Korea National Health and Nutrition Examination Survey (KNHANES) is financially supported by the Health Promotion Fund with administrative support by the Ministry of Health and Welfare.

\section{AUTHOR CONTRIBUTION}

Conceptualization: Kim SA. Methodology: Kim SA, Kim JY. Formal analysis: Heo NH, Park SH. Funding acquisi- 
tion: None. Project administration: Kim SA. Visualization: Kim YT, Oh KY, Park SH, Kim JY. Writing - original draft: Park ND, Kim SA. Writing - review and editing: Kim SA. Approval of final manuscript: all authors.

\section{REFERENCES}

1. Remelli F, Vitali A, Zurlo A, Volpato S. Vitamin D deficiency and sarcopenia in older persons. Nutrients 2019;11:2861.

2. Abiri B, Vafa MR. Vitamin D and sarcopenia. Adv Obes Weight Manag Control 2017;6:00155.

3. Chung E, Mo H, Wang S, Zu Y, Elfakhani M, Rios SR, et al. Potential roles of vitamin $\mathrm{E}$ in age-related changes in skeletal muscle health. Nutr Res 2018;49:23-36.

4. Semba RD, Blaum C, Guralnik JM, Moncrief DT, Ricks MO, Fried LP. Carotenoid and vitamin E status are associated with indicators of sarcopenia among older women living in the community. Aging Clin Exp Res 2003;15:482-7.

5. Khor SC, Abdul Karim N, Ngah WZ, Yusof YA, Makpol S. Vitamin E in sarcopenia: current evidences on its role in prevention and treatment. Oxid Med Cell Longev 2014;2014:914853.

6. Rondanelli M, Faliva MA, Peroni G, Moncaglieri F, Infantino V, Naso M, et al. Focus on pivotal role of dietary intake (diet and supplement) and blood levels of tocopherols and tocotrienols in obtaining successful aging. Int J Mol Sci 2015;16:23227-49.

7. Niki E. Role of vitamin E as a lipid-soluble peroxyl radical scavenger: in vitro and in vivo evidence. Free Radic Biol Med 2014;66:3-12.

8. Lukaski HC. Vitamin and mineral status: effects on physical performance. Nutrition 2004;20:632-44.

9. Servais S, Letexier D, Favier R, Duchamp C, Desplanches D. Prevention of unloading-induced atrophy by vitamin E supplementation: links between oxidative stress and soleus muscle proteolysis? Free Radic Biol Med 2007;42:627-35.

10. Aragno M, Mastrocola R, Catalano MG, Brignardello E, Danni O, Boccuzzi G. Oxidative stress impairs skeletal muscle repair in diabetic rats. Diabetes 2004;53:10828.

11. Ble A, Cherubini A, Volpato S, Bartali B, Walston JD, Windham BG, et al. Lower plasma vitamin $\mathrm{E}$ levels are associated with the frailty syndrome: the InCHIANTI study. J Gerontol A Biol Sci Med Sci 2006;61:278-83.

12. Bo Y, Liu C, Ji Z, Yang R, An Q, Zhang X, et al. A high whey protein, vitamin $\mathrm{D}$ and $\mathrm{E}$ supplement preserves muscle mass, strength, and quality of life in sarcopenic older adults: a double-blind randomized controlled trial. Clin Nutr 2019;38:159-64.

13. Scott W, Stevens J, Binder-Macleod SA. Human skeletal muscle fiber type classifications. Phys Ther 2001;81:1810-6.

14. Cerullo F, Gambassi G, Cesari M. Rationale for antioxidant supplementation in sarcopenia. J Aging Res 2012;2012:316943.

15. Nilwik R, Snijders T, Leenders M, Groen BB, van Kranenburg J, Verdijk LB, et al. The decline in skeletal muscle mass with aging is mainly attributed to a reduction in type II muscle fiber size. Exp Gerontol 2013;48:492-8.

16. Deschenes MR. Effects of aging on muscle fibre type and size. Sports Med 2004;34:809-24.

17. Jeon Y, Choi J, Kim HJ, Lee H, Lim JY, Choi SJ. Sex- and fiber-type-related contractile properties in human single muscle fiber. J Exerc Rehabil 2019;15:537-45.

18. Trappe S, Gallagher P, Harber M, Carrithers J, Fluckey J, Trappe T. Single muscle fibre contractile properties in young and old men and women. J Physiol 2003;552(Pt 1):47-58.

19. Staron RS, Hagerman FC, Hikida RS, Murray TF, Hostler DP, Crill MT, et al. Fiber type composition of the vastus lateralis muscle of young men and women. J Histochem Cytochem 2000;48:623-9.

20. Claflin DR, Larkin LM, Cederna PS, Horowitz JF, Alexander NB, Cole NM, et al. Effects of high- and lowvelocity resistance training on the contractile properties of skeletal muscle fibers from young and older humans. J Appl Physiol (1985) 2011;111:1021-30.

21. Institute of Medicine. Dietary reference intakes for vitamin C, vitamin E, selenium, and carotenoids. Washington, DC: National Academies Press; 2000. p. 186226.

22. Ford ES, Sowell A. Serum alpha-tocopherol status in the United States population: findings from the Third National Health and Nutrition Examination Survey. Am J Epidemiol 1999;150:290-300. 